\title{
Controlled Type -II Diabetes Mellitus" - No More Contra-Indication for Delayed Loading of Two Piece Dental Implants - A Case Report
}

\author{
Alekhya Ayalapuram ${ }^{1 *}$ and Pragya Jha ${ }^{2}$ \\ ${ }^{1}$ Consultant Implantologist and Periodontist, Apollo White Dental, Chennai \\ ${ }^{2}$ Post Graduate, Department Of Periodontics, Army College Of Dental Sciences, Secunderabad
}

Received: March 30, 2019; Accepted: April 16, 2019; Published: April 24, 2019

*Corresponding author: Dr. Alekhya Ayalapuram, University of Apollo White Dental, Undertaking Of Apollo Health And Lifestyle Limited, Tansi Nagar, 15th street, Velachery, Chennai, Tamil Nadu, Fax : 600042; Email : ayalapuram.alekhya@yahoo.in; Phone Number : 8019989867.

\begin{abstract}
With changing lifestyle and increasing obesity, prevalence of type -Il Diabetes Mellitus is increasing in geriatric individuals, who are generally prone to tooth loss. As a result the demand for implants in these patients is also increasing and planning implants has been a challenge to present day dentists. Diabetes Mellitus causes impaired metabolism in general, especially bone metabolism resulting in impaired Osseointegration and poor wound healing. Growing demand of implants in Type II DM patients has initiated research towards implants survival rates. Extensive research till now states - poorly controlled diabetic patients have higher implant failure rates, where as Diabetic patients with controlled blood glucose levels respond to implants in similar way as healthy patients. The present article is one such trail to confirm that type II Diabetes Mellitus is no more contra-indication for Implants till Blood glucose levels are controlled to normal levels.
\end{abstract}

Key words: Type-II Diabetes Mellitus; Two-piece implant; Delayed loading;

\section{Introduction}

Diabetes Mellitus (DM) is defined by the World Health Organization as "a metabolic disorder with heterogeneous etiologies that is characterized by chronic hyperglycemia and disturbances of carbohydrate, fat, and protein metabolism resulting from defects in insulin secretion, insulin action, or both [1]. Diabetic patients have increased frequency of periodontitis, tooth loss, and delayed wound healing [2-3].Today, dental implants are one of the prosthetic methods to replace missing teeth [5-6]. Dental implants represents an essential role in the rehabilitation of function, stability and aesthetics of the human dentition. With evolutionary advances in surgical protocols, implant design, and surface characteristics, Implants are considered as a secure and highly predictable procedure with a mean survival rate of $94.6 \%$ and a mean success rate of $89.7 \%$ after more than 10 years [4]. However, certain risk factors can predispose individuals to lower rates of success - for example smoking, a history of periodontitis, genetic traits and uncontrolled diabetes mellitus.

Controversially discussed as a relative contraindication for implant surgery is Diabetes. As the number of patients suffering from diabetes increases with age, there are more diabetic patients demanding implants due to tooth loss. Implant procedures are considered safe when diabetic levels are well controlled. Predictable complications in controlled diabetics are also found similar to healthy patients. Implant success is also improved by supportive administration of antibiotics and chlorhexidine.

\section{Materials and Methods}

A 58 years old male diabetic patient has come to Army College of Dental Sciences, Secunderabad, Telangana. With a chief complaint of missing lower right molar and need replacement with fixed permanent tooth. On medical history, he has type II Diabetes Mellitus since 10 years and was under medication. Dental history reveals that the tooth was extracted due to dental caries 3 years back. After clinical and radiographic examination, patient's HbA1c level was checked. On investigation, HbA1c levels were less than 6 - revealing controlled Diabetes Mellitus.

\section{INTRA-OPERATIVE PHOTOGRAPHS}

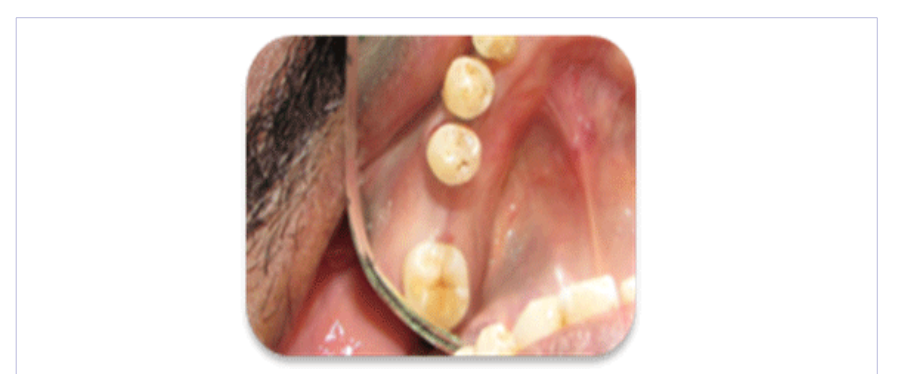

Figure 1: Crestal Incision

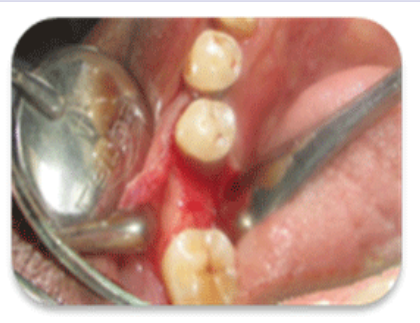

Figure 2: Full Thickness Flap Reflection 


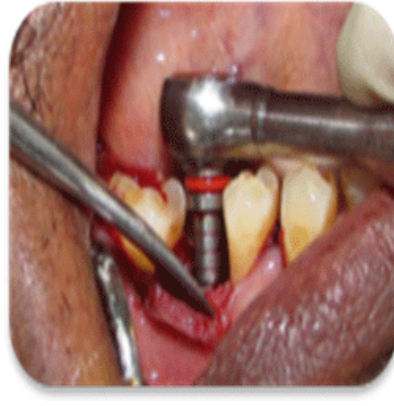

Figure 3: Wrenching Implant

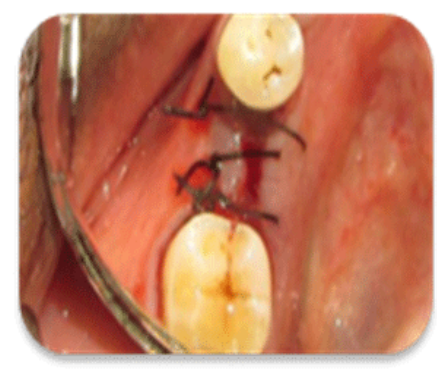

Figure 5: Interrupted Sutures Placed

A two-staged implant surgery with delayed loading was planned with a two-piece implant. Patient is advised antibiotic prophylaxis and $0.2 \%$ chlorhexidine mouth wash prior to implant surgery. Procedure is performed under strict aseptic conditions and sterile instruments Local aneasthesia is administered with $2 \%$ lignocaine with adrenaline (1:80000) through inferior alveolar nerve block. Full thickness flap is elevated and implant position is indexed and desired length is drilled with pilot drill, further drills were continued with one size less than the actual implant size, last drill is used for only coronal preparation. Implant is fixed and primary implant stability of $>30$ torque was checked. Flap is approximated with interrupted sutures and Coepack is placed.

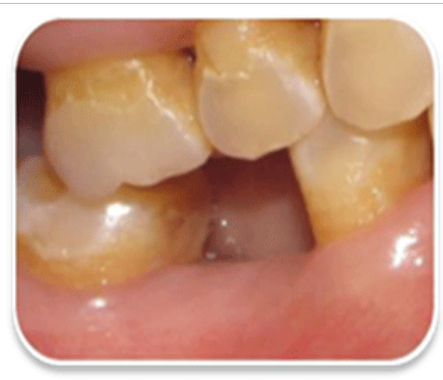

Figure 7: Pre-Operative
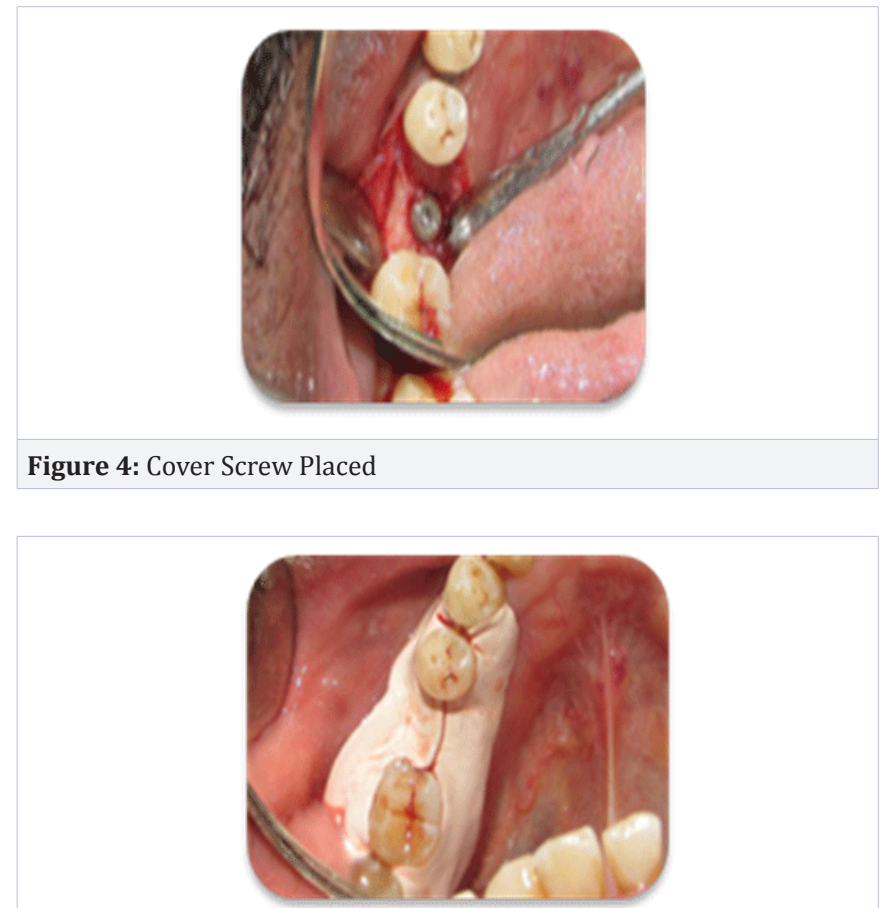

Figure 6: Coe-Pack Placed

Sutures are removed one week after surgery and checked for soft tissue healing which is eventful. 1:1 dilution of $0.2 \%$ chlorhexidine is prescribed for chemical plaque control.

After 3 months, second stage surgery is performed by removal of punch crestal tissue over implant and gingival former is placed. After 14 days, putty impression is taken with impression post in relation to 46 and prosthetic crown was cemented with straight abutment screwed on implant.

\section{Clinical Evaluation}

On clinical evaluation the implant is stable and functional with no signs of bleeding, mobility and healthy gingiva.

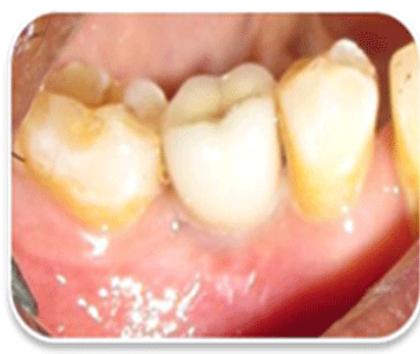

Figure 8: Post-Operative 


\section{Radiographic Evaluation}

A healthy mandibular bone has cortical type topography with a regular trabecular pattern on radiographic examination, the completely healed socket with regular trabeculae was evident in the pre-operative radiograph. Whereas 4 months post-operative radiograph reveal fully osseointegrated implant apically and partially integrated coronally. However the post-restorative radiograph after one year of cementation reveals increased radioopacity coronally stating fully osseointegrated implant with functional prosthesis.

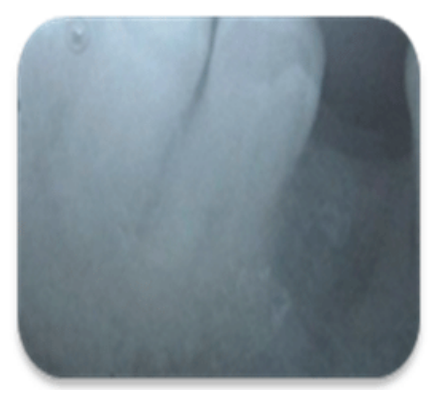

Figure 9: Pre-Operative

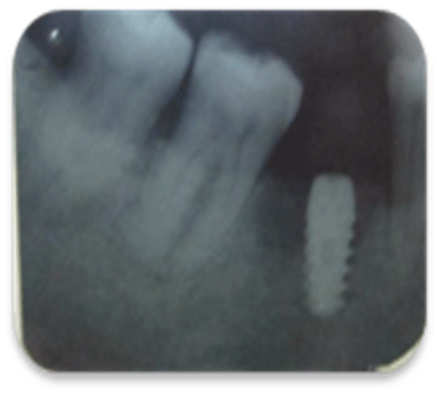

Figure 10: 4 months Post-Operative

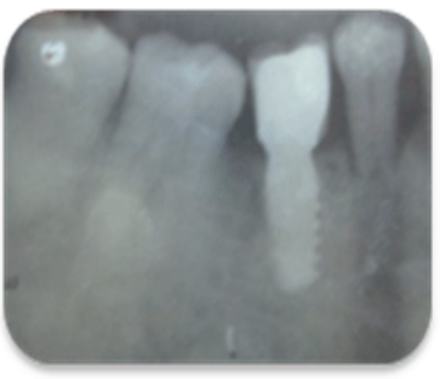

Figure 11: One year Post-Restorative

\section{Discussion}

On prosthetic crown cementation with proper occlusal table of a two piece implant (after second stage), the implant is fully functional with equal distribution of occlusal load. Thus the result from present case states that in normoglycemic type-II DM patients, implants gets osseointegrated with adequate secondary stability and responds similar to an healthy individual.
As most of implant failures occur due to patient's systemic and local factors within an year after restoration, the functional one-year follow-up in this case reveal type-II DM patient with controlled blood glucose levels respond as healthy individual.

On Review of literature, there is only a single survey showing marginally significant implant failures in type-II diabetic patients than healthy individuals [7]. However, most of the studies cannot give statistically significant difference in the survival rates of the implant treatment between well-controlled diabetics and nondiabetics. The recent systematic review did not find statistical difference between diabetic and non-diabetic patients by using a statistic meta-analytic approach on the effects of diabetes mellitus on implant failure rates [8].

An increased risk of early implant loss was observed in a retrospective study by Moy et al [9]. These authors reported that implant failure was threefold more likely in well-controlled DM patients than in healthy controls and that it occurred in the first few months but also continued over the following 10 years. In contrast, Tawil et al comparing in a prospective clinical study, 45 DM patients with 45 non-DM patients for a total of 499 dental implants followed for 1 to 12 years, did not find any statistically significant differences in the overall survival rate, that was independent from age, sex, diabetes duration, smoking, type of hypoglycemic therapy, or surgical approach [10].

\section{Conclusion}

Tooth replacement with implants has evolved and being chosen by patients in specific because of the conservative approach and improved masticatory efficiency. Still there are certain systemic and local risk factors which determine implant survival. One among them is most commonly faced Type-II Diabetes Mellitus.

Geriatric patients who are more prone to tooth loss have increased prevalence of type-II DM with age. Extensive studies and research revealed Type -II DM is never a contra-indication till it's controlled. Hence type-II DM patients can undergo implant procedure by following precautionary measures like:

- Delayed loading

- Improved oral hygiene,

- Frequent recall follow-ups,

- Regular monitoring of blood glucose levels and

- Controlling blood glucose level by regular Diabetologist's consultation.

\section{References}

1. World Health Organization. Definition, diagnosis and classification of diabetes mellitus and its complications: report of a WHO consultation. Part 1, Diagnosis and classification of diabetes mellitus: World Health Organization. 1999.

2. Khader YS, Dauod AS, El-Qaderi SS, Alkafajei A, Batayha WQ. Periodontal status of diabetics compared with non diabetics: a metaanalysis. J Diabetes Complications. 2006;20(1):59-68. 
3. Abiko Y, Selimovic D. The mechanism of protracted wound healing on oral mucosa in diabetes. Review. Bosn J Basic Med Sci.2010;10(3):186191.

4. Moraschini V, Poubel LA, Ferreira VF, Barboza Edos S. Evaluation of survival and success rates of dental implants reported in longitudinal studies with a follow-up period of at least 10 years: a systematic review. Int J Oral Maxillofac Surg. 2015;44(3):377-388. doi: 10.1016/j. ijom.2014.10.023

5. Mecall RA, Rosenfeld AL. Influence of residual ridge resorption patterns on implant fixture placement and tooth position.1.Int J Periodontics Restorative Dent.1991;11(1):8-23.

6. Schropp L, Wenzel A, Kostopoulos L, Karring T. Bone healing and soft tissue contour changes following single-tooth extraction:a clinical and radiographic 12-month prospective study. Int J Periodontics Restorative Dent. 2003;23(4):313-323.
7. Olson JW, Shernoff AF, Tarlow JL, Colwell JA, Scheetz JP, Bingham SF. Dental endosseous implant assessments in a type 2 diabetic population:a prospective study.Int. J. Oral Maxillofac.Implants. 2000; 1; 5(6):811-818.

8. Chrcanovic BR, Albrektsson T, Wennerberg A. Diabetes and oral implant failure: A systematic review. J Dent Res. 2014;93:859-67.

9. Moy PK, Medina D, Shetty V, Aghaloo TL. Dental implant failure rates and associated risk factors. Int J Oral Maxillofac Implants. 2005;20:569-577.

10. Tawil G, Younan R, Azar P, Sleilati G. Conventional and advanced implant treatment in the type II diabetic patient: Surgical protocol and long-term clinical results. Int J Oral Maxillofac Implants. 2008;23(4):744-52. 